\title{
Well-Balanced WENO Scheme for Tidal Wave Equations
}

\author{
Qiangqiang Zhu' ${ }^{1}$, Wei Sun², Jicai Zhang ${ }^{3}$ and Xianqing $\mathrm{Lv}^{1, *}$ \\ ${ }^{1}$ Key Laboratory of Physical Oceanography, Ocean University of China, Qingdao 266003, China; \\ ${ }^{2}$ College of Oceanic Atmospheric Sciences, Ocean University of China, Qingdao 266003, China; \\ ${ }^{3}$ Institute of Physical Oceanography Ocean College, Zhejiang University, Zhoushan 316021, China; \\ *Corresponding author
}

\begin{abstract}
In this paper, we investigate the high order wellbalanced essentially non-oscillatory (WENO) finite difference scheme for simulations of tidal wave equations. The third order WENO scheme is employed to capture high gradients in an essentially non-oscillatory manner with a discretization by the third order TVD Runge-Kutta method. In this study, several one(1D) and two- dimensional (2D) numerical cases are carried out in comparison with a central difference scheme (CDS). The results demonstrate the the exact conservation property (Cproperty), high order accuracy and essentially non-oscillatory shock capturing of the smooth and discontinuous solutions respectively.
\end{abstract}

Keywords-high order; well-balanced; WENO; tidal wave equations; source term

\section{INTRODUCTION}

The tidal wave equations are regarded as an important model in fluid dynamics which play an important role in investigating other processes. [10, 13]. There have been a lot of efforts devoted to the numerical scheme. Fang and Yang [4] applied the central finite difference scheme to model the tides and tidal currents of the m1, M2, M4 tidal constituents in the Bohai Sea. Afterwards, the finite-volume costal ocean model (FVCOM) [3] are applied to obtain accurate solutions based on unstructured grids, but to produce a large amount of computational cost. Meanwhile, most of the numerical schemes can not meet high order accuracy either efficiency while the solutions might exhibit a finite time singularity due to the complex topography or an discontinuous initial condition. Therefore, accurate and stable solutions have received much attention. Liu et al. [8] firstly developed the ENO scheme [5] by proposing the weighted ENO scheme (WENO) as a wellknown highorder and shock capturing scheme. Jiang and Shu [7] developed the WENO scheme (WENO-JS) via improving the smoothness measurement of a numerical solution. To maintain the exact conservation property and obtain high order accuracy, WENO schemes with a balance (well-balanced WENO) were proposed [11]. Later, Later, Xing and Shu [12] proposed high order well-balanced WENO-JS scheme that achieved genuine fifth order accuracy for the general solutions. Furthermore, Castro et al. [2] proposed the improved WENO finite difference scheme (WENO-Z) scheme which can obtain genuine solutions of the shallow water system with discontinuities, which is also less dissipative and higher resolution than the WENO-JS scheme for a larger class of problems.
The purpose of this study is to investigate its conservative property capturing high order accuracy and high gradient in comparison with a central finite difference scheme. The paper is organized as follows. In section 2 , a very brief introduction to the well-balanced WENO-Z scheme for solving the tidal wave equations will be given. Section 3 contains several oneand two-dimensional cases, which are conducted to verify the satisfaction of the essentially non-oscillatory property and high order accuracy of the WENO-Z scheme. Conclusions are given in section 4.

\section{THIRD ORDER WELL-BALANCED WENO SCHEME}

The two-dimensional tidal wave equations in the Cartesian domain can be written compactly as:

$$
\boldsymbol{Q}_{t}+\nabla \cdot \overrightarrow{\boldsymbol{F}}=\boldsymbol{S}
$$

$\boldsymbol{Q}, \overrightarrow{\boldsymbol{F}}=(F, G)$ are the conservative variables, flux vector, and source terms respectively, or, explicitly,

$$
\begin{aligned}
& \boldsymbol{Q}=(H, H u, H v)^{T}, \\
& \boldsymbol{F}=\left(H u, H u^{2}+1 / 2 g H^{2}, H u v\right)^{T}, \boldsymbol{G}=\left(H v, H u v, H v^{2}+1 / 2 g H^{2}\right)^{T}, \\
& \boldsymbol{S}=\left(0, f H v-g H b_{x}-k u \sqrt{u^{2}+v^{2}},-f H u-g H b_{y}-k v \sqrt{u^{2}+v^{2}}\right)^{T},
\end{aligned}
$$

where the superscript ${ }_{T}$ denotes the transpose, $t$ is time, $x$ and $y$ are Cartesian coordinates, $H$ denotes the total water depth related to time evolution, $u$ and $v$ are the velocity components to the east and north, $b$ is the bottom topography, $f$ is the Coriolis parameter, $k$ is the bottom friction coefficient, and $g=9.812 \mathrm{~m} / \mathrm{s}^{2}$ is the gravitational constant.

Take the approximation of the derivative of flux term $F_{x}$ in (1) as an example to describe the reconstruction procedure of the third order WENO-Z scheme. Consider an equidistant grid defined by the points $x_{i}=i \Delta x, i=0, \cdots, N$, which arecalled cell centers, with cell boundaries given by $x_{i+\frac{1}{2}}=x_{i}+\frac{\Delta x}{2}$, where $\Delta x$ is the uniform grid spacing (see Fig.1). 


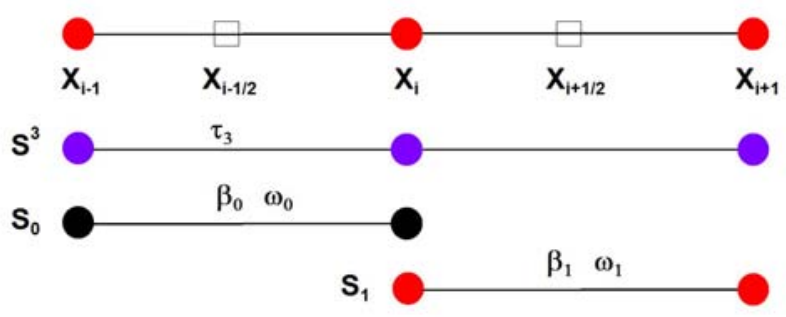

FIGURE I. THE 3-POINTS STENCIL $S^{3}$ (COMPOSED OF THREE 3POINTS SUBSTENCILS $\left\{S_{0}, S_{1}\right\}$ ), CLASSICAL SMOOTHNESS INDICATORS $\left\{\beta_{0}, \beta_{1}\right\}$, AND NONLINEAR WEIGHTS $\left\{\omega_{0}, \omega_{1}\right\}$, AND A NEW SMOOTHNESS INDICATOR $\tau_{3}$ USED IN THE STENCIL $S^{3}$

The semi-discretized form of hyperbolic parts of (1) is transformed into a system of ordinary differential equations and solved by the method of lines,

$$
\frac{d Q_{i}(t)}{d t}=-\left.\frac{\partial F}{\partial x}\right|_{x=x_{i}}, i=0, \cdots, N
$$

where numerical value $Q_{i}(t)=Q\left(x_{i}, t\right)$.

Here, we describe the third order nonlinear adaptive polynomial reconstruction process. To form the difference expression of the flux across the uniformly spaced cells and to obtain high-order numerical flux consistent with the hyperbolic conservation laws, a conservative finite difference formulation is required at the cell boundaries. By defining a numerical flux function $\bar{F}(x)$ implicitly, one has

$$
F(x)=\frac{1}{\Delta x} \int_{x-\frac{\Delta x}{2}}^{x+\frac{\Delta x}{2}} \bar{F}(\xi) d \xi
$$

such that the spatial derivative in (3) is approximated by a conservative finite difference formula at the cell center $x_{i}$,

$$
\frac{d Q_{i}(t)}{d t}=-\frac{1}{\Delta x}\left(\bar{F}_{i+\frac{1}{2}}-\bar{F}_{i-\frac{1}{2}}\right)
$$

where $\bar{F}_{i \pm \frac{1}{2}}=\bar{F}\left(x_{i \pm \frac{1}{2}}\right)$. High order polynomial interpolations to $\bar{F}_{i \pm \frac{1}{2}}$ are computed using known values $F_{j}=F\left(x_{j}\right), j=i-1, \cdots, i+1$.

As shown in the Fig.1, the 3-points global stencil $S^{3}$ is subdivided into two 2-points substencils $\left\{S_{0}, S_{1}\right\}$. Then the third degree polynomial approximation $\hat{F}_{i \pm \frac{1}{2}}=\bar{F}_{i \pm \frac{1}{2}}+O\left(\Delta x^{3}\right)$ can be built through a convex combination of three first degree interpolation polynomials $\hat{F}^{k}(x)$ in substencils $S_{k}, k=0,1$ at the cell boundaries $x_{i \pm \frac{1}{2}}$,

$$
\hat{F}_{i \pm \frac{1}{2}}=\sum_{k=0}^{1} \omega_{k}^{ \pm} \hat{F}^{k}\left(x_{i \pm \frac{1}{2}}\right)
$$

where $\omega_{k}$ are the normalized nonlinear weights (which will be described below) and

$$
\hat{F}^{k}\left(x_{i+\frac{1}{2}}\right)=\sum_{j=0}^{1} c_{k j} F_{i-k+j}, \quad i=0, \cdots, N,
$$

with Lagrangian interpolation coefficients $c_{k j}$ [1].

The regularity of the interpolation polynomial approximation $\hat{F}^{k}(x)$ of the substencil $S_{k}$ at $x_{i}$ is measured by the local lower order smoothness indicators $\beta_{k}$, which are given by the explicit expression of the smoothness indicators $\beta_{k}$ can be found in [7].

In the WENO-Z finite difference scheme ([2]), the nonlinear weights $\omega_{k}^{z}$ are defined as

$$
\omega_{k}^{z}=\frac{\alpha_{k}^{z}}{\sum_{l=0}^{2} \alpha_{l}^{z}}, \quad k=0,1,
$$

where

$$
\alpha_{k}^{z}=d_{k}\left(1+\left(\frac{\tau_{3}}{\beta_{k}+\varepsilon}\right)^{p}\right)
$$

Here $\tau_{3}=\left|\beta_{1}-\beta_{0}\right|$, which has a leading truncation error of order $O\left(\Delta x^{3}\right)$.

To maintain the exact C-property, we follow the WENO-LF reconstruction procedures proposed in [12] to preserve a delicate balance of the flux and the source terms in the steady state. Details can be referred to [12].

\section{NUMERICAL RESULTS}

To demonstrate the good performance of the well-balanced WENO-Z finite difference scheme, $1 \mathrm{D}$ and 2D numerical tests were studied. We shall provide both the numerical solutions computed by WENO-Z scheme and CDS scheme. The third order TVD Runge-Kutta method [2] with $C F L=0.45$ is always used for the temporal evolution of the resulted ODE at each grid points.

\section{A. A Small Perturbation of a 1D Steady-State Water}

This case is to verify the shock capturing property of the WENO-Z scheme for a rapidly varying flow over a smooth bottom and a perturbation of a stationary state. We follow the 
typical 1D quasi-stationary test case given in [12]. The bottom topographies $b(x)$ consists of a hump as follows

$$
b(x)=\left\{\begin{array}{cc}
\frac{(\cos (10 \pi(x-1.5))+1)}{4}, & \text { if } 1.4 \leq x \leq 1.6 \\
0, & \text { otherwise. }
\end{array}\right.
$$

The corresponding initial conditions are given as follows,

$$
\begin{aligned}
& h(x, 0)=\left\{\begin{array}{cc}
1-b(x)+\xi, & \text { if } 1.1 \leq x \leq 1.2, \\
1-b(x), & \text { otherwise, }
\end{array},\right. \\
& u(x, 0)=0,
\end{aligned}
$$

where $\xi$ is a nonzero constant amplitude of the perturbation. Here the case with a big pulse $\xi=0.2$ has been simulated with both the WENO-Z scheme and CDS scheme.
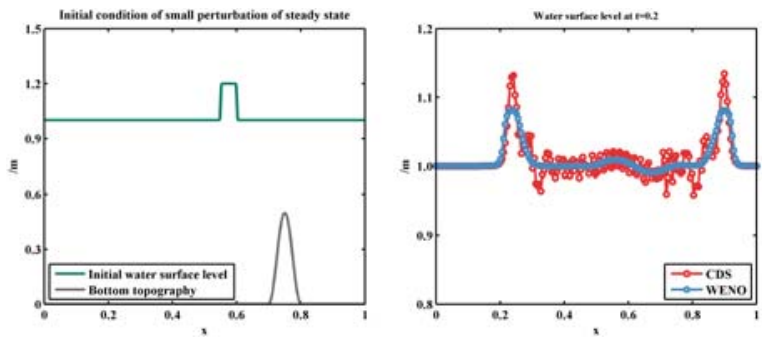

FIGURE II. (LEFT) INITIAL WATER SURFACE LEVEL $H+b$ AND BOTTOM TOPOGRAPHY $b$, (RIGHT) WATER DISCHARGE $H u$ AT TIME $t=0.2$ AS COMPUTED BY THE WENO-Z SCHEME AND CDS SCHEME.

Once encountered a perturbation in the center of the steady state water, there generates a convergence that induces a gravity wave finally. Driven by pressure gradient force, the small disturbance will be split into two smaller waves propagating to the left and the right respectively. The solutions computed by the two schemes are shown in Fig.2, where it is obviously that the CDS scheme produce large spurious numerical oscillations while the WENO-Z scheme obtained smooth solutions. Meanwhile, the small traveling waves with the initial pulse perturbations are already well resolved by the WENO-Z scheme and passed over the bump, which verify the essentially nonoscillatory property of the WENO-Z scheme while capture the high gradient.

\section{B. 1D Simulation of Tidal Wave Flow Over a Channel}

In this case we compare performances of the two schemes in smooth initial condtion. Here we follow the example presented in [12] where an almost exact solution was given. The bottom is given as

$$
b(x)=10+\frac{40 x}{L}+10 \sin \left(\pi\left(\frac{4 x}{L}-\frac{1}{2}\right)\right),
$$

where $L=14000 m$ is the channel length. Especially if we set the initial and boundary condition as follows,

$$
\begin{aligned}
& h(x, 0)=60.5-b(x), \quad(h u)(x, 0)=0, \\
& h(0, t)=64.5-4 \sin \left(\pi\left(\frac{4 t}{86400}+\frac{1}{2}\right)\right), \\
& (h u)(L, t)=0,
\end{aligned}
$$

Then we can obtained a very accurate approximate solution based on asymptotic analysis presented in [12]. Here the bottom friction and Coriolis force can be ignored. We computed the equations until time $t=7552.13 \mathrm{~s}$ with an equidistant grid $\Delta x=70 \mathrm{~m}$, and the comparison of the results are shown in Fig.3. From the errors between the simulated and accurate solutions of water surface level $H$ and velocity $u$, it can be seen obviously that the WENO-Z scheme is more accurate than the CDS scheme, which verify the property of high order accuracy of the WENO-Z scheme.
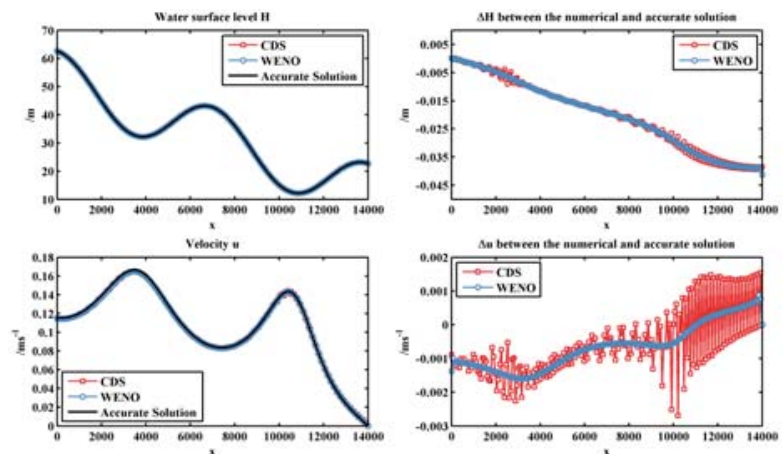

FIGURE III. NUMERICAL AND ANALYTIC SOLUTIONS (LEFT) AND NUMERICAL ERRORS (RIGHT) FOR THE TIDAL WAVE FLOW. TOP: WATER HEIGHT $H$; BOTTOM: VELOCITY $u$.

\section{2D Simulation of M2 Tidal Constituent in Rectangular Sea Area}

In this case, we design a idealised numerical experiment in rectangular sea area to achieve a simulation of the M2 tidal constituent. The computational area is set as a rectangular area $\left(117^{\circ} 30^{\prime} \mathrm{E}-126^{\circ} 30^{\prime} \mathrm{E}, 27^{\circ} \mathrm{N}-41^{\circ} \mathrm{N}\right)$. The horizontal resolution is $10^{\prime} \times 10^{\prime}$. The initial water depth and the corresponding bottom friction coefficient are set as $H=50$ and $k=0.0012$, respectivly. The bottom topography is a Gaussian function depicted as follow,

$$
b(i, j)=b_{\max } e^{\frac{(i-42)^{2}+(j-21)^{2}}{2 \sigma^{2}}}
$$

where $i$ and $j$ are grid indexes along the longitude and latitude respectively ( $1 \leq i \leq 42,1 \leq j \leq 84$ ), parameter is $b_{\max }=10$. In this example, the time-dependent OBs along $34^{\circ} N$ at $j$ th step are given as follows,

$$
\zeta_{m_{l}, n_{l}}^{j}=a_{l} \cos \left(\omega j \Delta t+\theta_{l}\right)
$$

where $m_{l}$ and $n_{l}$ are grid indexes, $\omega$ is frequency of M2 constituent, $\Delta t$ is time step. The amplitude $a_{l}$ (constant), 
phase $\theta_{l}$ (a linear function) and spatial distribution of initial OBs related to the indexes are shown in Fig.4.
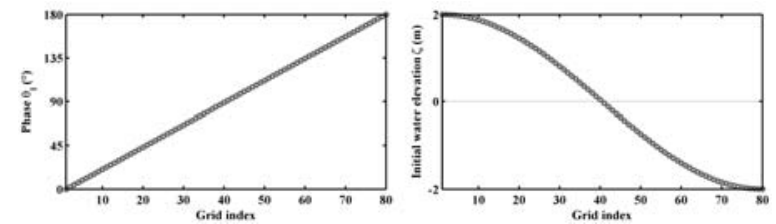

FIGURE IV. THE SPATIAL DISTRIBUTION OF PHASE $\theta_{l}$ (LEFT) AND INITIAL OBS (RIGHT)

Once given a elevation force at every step along $34^{\circ} \mathrm{N}$, naturally, the formed gravitational wave then induces internal divergence with tidal energy spreading to induce water fluctuation. We carried out the simulation using the WENO-Z scheme and CDS scheme, and the comparison of the cotidal charts obtained by harmonic analysis [6,9] of the two schemes are presented in Fig.5.
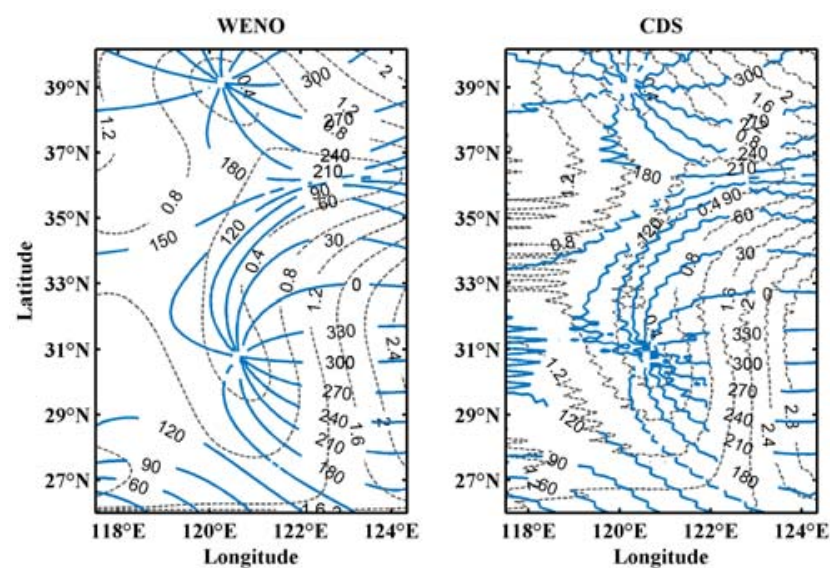

FIGURE V. THE COTIDAL CHARTS OF M2 TIDAL CONSTITUENT IN RECTANGULAR SEA AREA OBTAINED BY THE WENO-Z

SCHEME (LEFT) AND CDS SCHEME (RIGHT) RESPECTIVELY, WHERE DASHED LINE DENOTES THE AMPLITUDE (M), SOLID LINE DENOTES THE PHASE (DEGREE).

As shown in Fig.5, there generated two tide-amphidromic points due to the superposition by incident wave and reflected wave. Furthermore, as a result of the topographic effect, the tide-amphidromic points are generated almost in small gradient area. Meanwhile, the cotidal charts obtained by the CDS scheme produced a large amount of oscillation although the locations of the tide-amphidromic points are nearly same as the WENO-Z scheme, while the results of the WENO-Z scheme are quite smooth. The cotidal charts obtained by WENO-Z with essentially free of oscillation reflect the practical wave propagation of M2 tidal constituent in rectangular sea area, which further verify its high order accuracy.

\section{CONCLUSION}

In this study, a third order well-balanced WENO-Z finite difference scheme is successfully applied to solving the tidal wave equations. The WENO-Z scheme achieves better performance in both $1 \mathrm{D}$ and $2 \mathrm{D}$ cases. $1 \mathrm{D}$ steady-state water case with discontinuous initial condition verify the essentially nonoscillatory property of the WENO-Z scheme, while the CDS scheme obviously shows its drawbacks due to the numerical oscillation generated in the process of calculation. In the simulation for the M2 tidal constituent in an ideal channel and rectangular sea area with smooth initial conditions, the cotidal charts obtained via harmonic analysis by WENO-Z scheme are all better than the results obtained by the CDS scheme. All the results demonstrate that the WENO-Z is superior to the CDS scheme in resolving smooth parts and capturing discontinuities. Furthermore, it is of great practical value to extend the WENO-Z scheme to simulate other ocean motions with high-gradient water elevation. Storm surge and Qiantang tidal bore constitute ongoing works.

\section{ACKNOWLEDGMENTS}

The authors would like to acknowledge the funding support of this research by the Natural Science Foundation of Zhejiang Province through Grant LY15D060001, the National High Technology Research and Development Program of China (863 Program) through grant2013AA122803, the National Science Foundation of Shandong Province, China through grant ZR2014DM017, the National Natural Science Foundation of China through grant 41371496 and 41206001.

\section{REFERENCES}

[1] D. Balsara and C.W. Shu. Monotonicity preserving weighted essentially nonoscillatory schemes with increasingly high order of accuracy. J. Comput. Phys., vol. 160, 2000, pp.405-452.

[2] M. Castro, B. Costa, and W.S. Don. High order weighted essentially nonoscillatory weno-z schemes for hyperbolic conservation laws. J. Comput. Phys., vol. 230, 2011, pp.1766-1792.

[3] C.S. Chen, H. Liu, and R.C. Beardsley. An unstructured grid, finitevolume, threedimensional, primitive equations ocean model: Application to coastal ocean and estuaries. J.Atmos.Oceanic.Technol., vol. 20, 2003, pp.159-186.

[4] G.H. Fang and J.F. Yang. A two-dimensional numerical model of tidal motions in the bohai sea. Chin. J. Ocean. Limnol., vol. 3, 1985, pp.136152

[5] Harten, B. Engquist, S. Osher, and S.R.Chakravarthy. Uniformly highorder accurate nonoscillatory schemes, i. SIAM J. Numer. Anal., vol. 24, 1997.

[6] L.H. Horn and R.A. Bryson. Harmonic analysis of the annual march of precipitation over the united states. Annals. Asso. Amer. Geogra., vol. 50, 1960, pp.157-171.

[7] G.S. Jiang and C.W. Shu. Efficient implementation of weighted eno schemes. J. Comput. Phys., vol. 126, 1996, pp.202-228.

[8] X.D. Liu, S. Osher, and T. Chan. Weighted essentially nonoscillatory schemes. J. Comput. Phys., 1994, pp.115-200.

[9] O.S. McGee. The use of the harmonic dial in rainfall climatology. South. Afri. Geogra. J., vol. 51, 1969, pp.65-72.

[10] W.H. Munk. Once again: once again-tidal friction. Prog. Ocean., vol.40, 1997, pp.7-35.

[11] S. Vukovic, N. Crnjaric-Zic, and L. Sopta. Weno schemes for balance laws with spatially varying flux. J. Comput. Phys., vol. 199, 2004, pp.87-109.

[12] Y.L. Xing and C.W. Shu. High order finite difference weno schemes with the exact conservation property for the shallow water equations. J. Comput. Phys., vol. 208, 2005, pp.206-227.

[13] J.C. Zhang and X.Q. Lu. Inversion of threedimensional tidal currents in marginal seas by assimilating satellite altimetry. Comput.Methods Appl.Mech.Engrg., vol. 199, 2010, pp.3126-3126. 